\title{
Oncocytoma Parotid Gland: A Case Report and B rief Review of Literature
}

\author{
${ }^{1}$ Sunil Kumar, ${ }^{2}$ As hwini Sethi, ${ }^{3}$ Satwinder Pal Singh, ${ }^{4}$ Vikas Sharma
}

\begin{abstract}
Very few cases of oncocytoma parotid have been reported in literature. Because of its rarity (less than $1 \%$ of salivary gland tumors) and clinical presentation of the tumor, there is a tendency among the clinicians to misdiagnose it as pleomorphic adenoma, hemangioma or other forms of oncocytosis. Only a histopathological examination can confirm it. We report here a case of oncocytoma of parotid gland in a 70-year-old male who had the tumor for over 9 years.
\end{abstract}

Keywords: O ncocytoma, Parotid, FNAC.

How to cite this article: Kumar S, S ethi A, Singh SP, Sharma V. Oncocytoma Parotid G land: A Case R eport and B rief R eview of Literature. Int J Head Neck Surg 2014;5(1):25-27.

\section{Source of support $\mathrm{Nil}$}

Conflict of interest None

\section{INTRODUCTION}

Salivary gland neoplasms represent the most complex and diverse group of tumors encountered by head and neck surgeons, their diagnosis and management is complicated by their relative infrequency ( $1 \%$ of head and neck tumors), the limited amount of pretreatment information available and wide range of biological behavior seen. ${ }^{1}$ Oncocytomas are rare tumors. They are predominantly tumors of those over the middle age. Women, usually in the seventh or eighth decade are likely to be affected. Parotid glands are by far the most frequent site. These tumors are slow growing and may rarely be bilateral. ${ }^{2}$

We present here a case of oncocytoma in a 70-year-old male who had a lemon-sized swelling in left parotid for about 9 years without undergoing any significant change in its size and character. Patient reported only for its unsightly appearance but had not any other complaints.

\section{CASE REPORT}

A 70-year-old male reported in EN T Outpatient D epartment with a mass near angle of mandible on left side for 9 years. It was a painless swelling not increasing in size for last many years. Patient had no other significant complaints. On

\footnotetext{
${ }^{1}$ S enior Resident, ${ }^{2,3}$ Assistant Professor, ${ }^{4}$ J unior Resident

${ }^{1-4}$ Department of ENT, Head and Neck Surgery, Army College of Medical Sciences, Delhi Cantt, New Delhi, India
}

Corresponding Author: Sunil Kumar, Senior Resident Department of ENT, Head and Neck Surgery, Army College of Medical Sciences, Delhi Cantt, New Delhi, India Phone: 919899028040, e-mail: angralsunil@ gmail.com examination, a solitary $03 \times 02 \mathrm{~cm}$ sized mass left parotid posterior to angle of mandible was present (Fig. 1). It was a nontender, nonfluctuant, nonpulsatile sweling, not adherent to overlying skin and underlying structures, margins were regular. No signs of facial palsy were seen. Fine needle aspiration cytology (FNAC) report was suggestive of oncocytic neoplasm. On contrast-enhanced computerized tomography (CECT), a rounded hyperdense lesion in the superficial lobe of left parotid was seen which according to radiologist could be benign parotid neoplasm, hemangioma or enlarged lymph node (Fig. 2). UItrasonography (USG) identified a $2.5 \times 1.7 \mathrm{~cm}$ sized lobulated solid mass in left parotid region which was closely abutting left parotid gland and was very vascular. Based on the above findings, a provisional diagnosis of oncocytoma parotid was made with differential diagnosis of hemangioma, and patient was worked up for superficial parotidectomy. Superficial parotidectomy was done with preservationn of facial nerve. Excised mass was sent for histopathological examination, which confirmed it as oncocytoma. Postoperative period was uneventful.

\section{PATHOLOGICAL FINDINGS}

Grossly, the resected specimen measured $5 \times 4 \times 2 \mathrm{~cm}$ in size. $M$ acroscopically, the tumor was nodular circumscribed lesion measuring $2 \times 1.5 \times 2 \mathrm{~cm}$ in size and brown homogenous in color. Microscopic findings show capsulated tumor composed of lobules of oncocytic cells. The cells were large round with abundant granular cytoplasm.

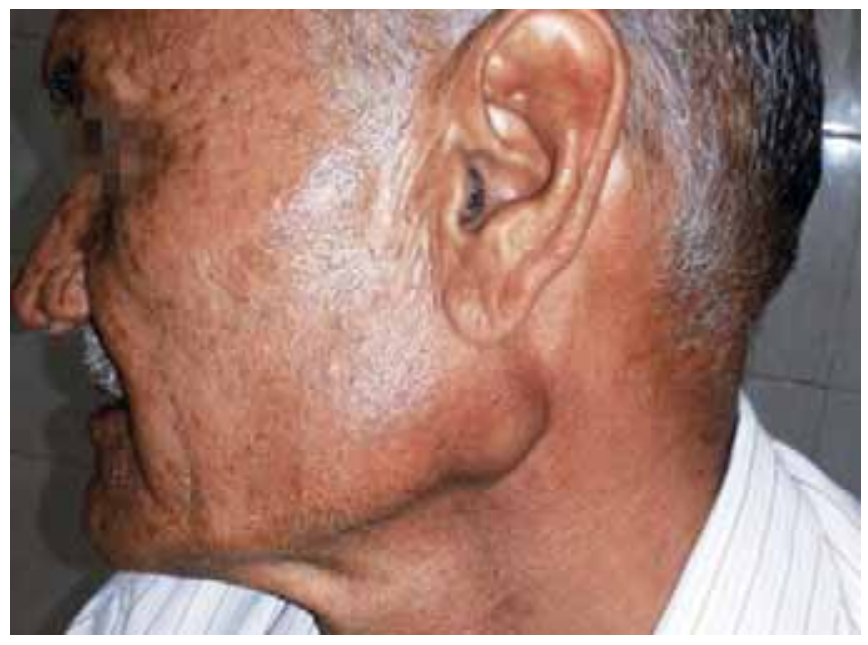

Fig. 1: Clinical photograph of the patient showing tumor left parotid 

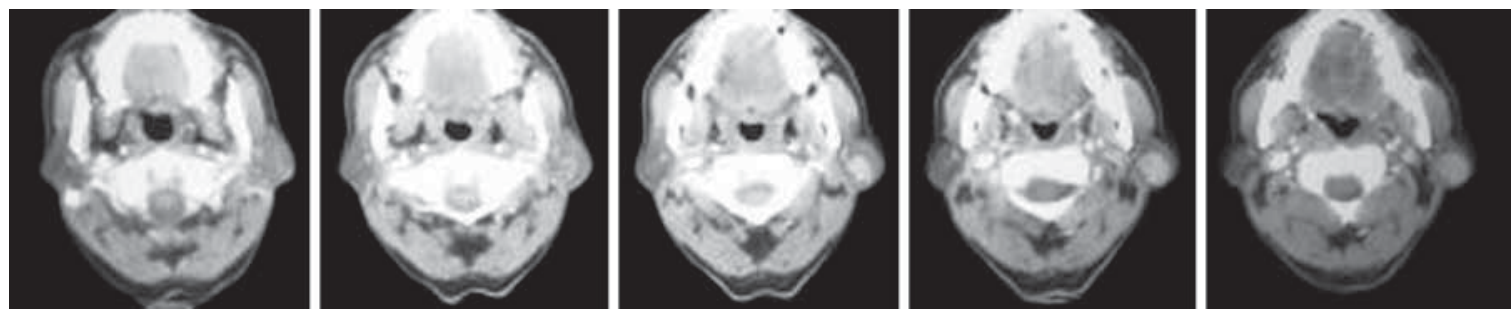

Fig. 2: CECT images showing tumor localized within the parotid tissue

No mitotic figure noted. No evidence of malignancy seen (Fig. 3). These features are consistent with oncocytoma.

\section{DISCUSSION}

Oncocytes are epithelial cells which appear as cells with abundant granular, eosinophilic cytoplasm, a centrally pyknotic nucleus and ultrastructurally are crammed with numerous mitochondria of various sizes. Oncocytes are seen in various organs like salivary glands, thyroid, parathyroid, pituitary, nasal cavities, sinuses, ocular caruncle, lacrimal glands, buccal mucosa, Eustachian tube, larynx, esophagus and organs like liver, pancreas and kidney. ${ }^{3,4}$ World Health Organization (WHO) classification of salivary gland neoplasms recognizes three oncocytic entities: oncocytosis, oncocytoma and oncocytic carcinoma. Oncocytomas are more common than oncocytic carcinomas. ${ }^{4}$

Oncocytomas usually occur in the elderly and affect the parotid glands in $80 \%$. Pathologically, oncocytoma is described as a well-circumscribed mass, composed of layers of oncocytes (small round nucleus, microgranular, eosinophilic cytoplasm). Pathogenesis is quite obscure, although mitochondrial functional defects are believed to mediate the progressive degeneration of the salivary epithelial cells. ${ }^{5}$ Of note, only one mitochondrial DNA rearrangement (among 200 described) has been linked to parotid tumorogenesis. ${ }^{6}$ Tandler et al ${ }^{7}$ reveal ed by electron microscopy that the oncocytes contained unusually large number of mitochondria. Oncocytic cells are thought of as

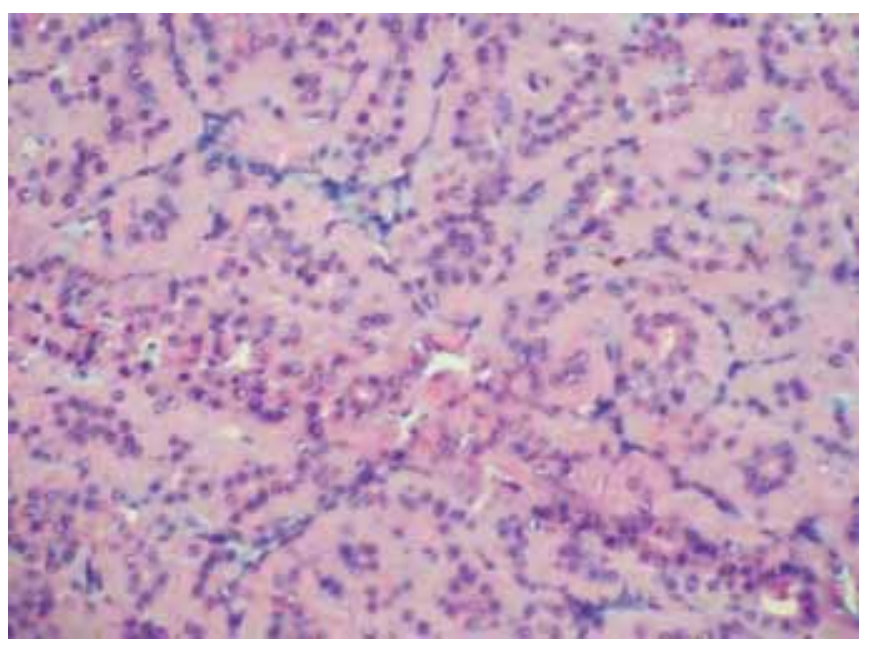

Fig. 3: Histopathological picture of typical features of oncocytic cells metaplastic cells formed in response to adverse changes, with the normal cells losing their original specialization. ${ }^{4}$ A ging is also thought to cause a functional exhaustion of mitochondrial enzymes, and a compensatory hyperplasia of mitochondria can occur, which in turn is responsible for the oncocytic change. Indeed, solitary oncocytes appear most often as incidental findings in aging salivary tissue, with studies showing up to $80 \%$ presence in persons older than 70 years of age. ${ }^{3}$ In our case also, the age of patient was 70 years which points tow ard the progressive degeneration of salivary epithelial which lead to oncocytic changes.

The correlation of certain viruses, such as EBV, HIV, HHV-8, HTLV-1 and HPV with parotid neoplasias has been documented. $V$ lachaki $E$ et al described the case of a 74-year-old female patient with left parotid oncocytoma and a previous history of immune thrombocytopenia and chronic HBV infection. ${ }^{8}$ In our case, there was no history of viral infection or thrombocytopenia in the patient.

A reas of oncocytic metaplasia can be seen in a host of salivary gland tumors like basal cell adenoma, pleomorphic adenoma, myoepithelioma, cystadenoma, canalicular adenoma, polymorphous low grade adenocarcinoma, Warthin's tumor, acinic cell carcinoma and mucoepidermoid carcinoma. ${ }^{4}$ However, oncocytes also give rise to neoplasms like oncocytomas and its malignant counterpart, the oncocytic carcinoma. Histopathological findings in our case are typical of a benign oncocytic neoplasm which points toward the fact that prolonged follow-up may not be necessary.

Fine needle aspiration is the procedure of choice for making a diagnosis in the majority of cases, although its sensitivity is reported to be only $29 \% .{ }^{5}$ Fine needle aspiration cytol ogy has increasingly been used as a primary screening tool for salivary gland lesions with high levels of sensitivity and specificity. However, as salivary glands are notorious for having overlapping morphological features, diagnosis by cytology alone often becomes difficult. ${ }^{2}$ The situation may slightly improve by using multiple passes from the swelling.

Diouf MS et al reported a case of oncocytoma of the left parotid gland in a 69-year-old woman in whom FNAC was for a pleomorphic adenoma. Through this case, they highlighted the importance of histopathology in the positive 
diagnosis of parotid oncocytoma as well as in its differential diagnosis and also the place of FNAC. ${ }^{9}$

In salivary glands, oncocytes may be present in a plethora of conditions ranging from hyperplasia to overtly malignant lesions. Diagnosis by FNAC may be very difficult due to focal sampling of the lesion, as oncocytic change can occur in a large variety of neoplastic as well as nonneoplastic conditions. Histopathology remains the gold standard to clinch the precise diagnosis. Chakrabarti I et al presented a case of a cytologically diagnosed oncocytic lesion with a possibility of oncocytoma. However, on subsequent histopathology, the lesion was diagnosed as diffuse hyperplastic oncocytosis. ${ }^{10}$ In our case, the FNAC was clearly suggestive of oncocytic features of the tumor and after histopathological examination oncocytoma was confirmed.

Surgical management with radical or superficial parotidectomy represents the cornerstone of therapy. ${ }^{5}$ In our case also, we removed the tumor by superficial parotidectomy and preserving the facial nerve postoperative period was uneventful.

\section{SUMMARY}

B ecause of the rarity of the lesions, oncocytomas are sometimes misdiagnosed as pleomorphic adenomas or wrongly reported as other forms of oncocytosis which are more common. Fine needle aspiration cytology is helpful in many cases to preoperatively diagnose the type of tumor; however, only histopathological examination can confirm the true nature. Ultrasonography is not much helpful in differentiating the benign from invasive tumors. M agnetic resonance imaging (M RI) and CECT are hel pful in planning the management. Surgical excision remains the cornerstone for treatment.

\section{REFERENCES}

1. Nagarkar N M , Bansal S, Dass A, Singhal SK, M ohan H. Salivary gland tumours- our experience. Indian J Otolaryngol Head N eck Surg 2004 Jan;56(1):31-34. doi: 10.1007/B F02968769

2. Palmer TJ, Gleeson M, Eveson JW, Cawson RA. Oncocytic adenomas and oncocytic hyperplasia of salivary glands: a clinicopathological study of 26 cases. Histopathology 1990;16:487-493.

3. KontaxisA, Zanarotti U, K ainz J, B eham A. Diffuse hyperplastic oncocytosis of the parotid gland. L aryngorhinootologie 2004;83: 185-188.

4. Prabakaran SS, Chen F, A guirre A. O ncocytoma of the parotid gland and its mimickers: a comprehensive review. NAJ M S 2010; 3:171-180.

5. Capone RB, Ha PK, Westra WH, Pilkington TM, Sciubba JJ, K och W M , Cummings CW. Oncocytic neoplasms of the parotid gland: a 16-year institutional review. Otolaryngol Head N eck Surg 2002;126:657-662.

6. L ewis PD, B axter P, Paul G riffiths A, Parry J M , Skibinski DO. Detection of damage to the mitochondrial genome in the oncocytic cells of Warthin's tumour. J Pathol 2000;191:274-281.

7. Tandler B. Fine structure of oncocytes in human salivary glands. Virchows A rch Pathol A nat Physiol K lin M ed 1966;341:317-226.

8. Vlachaki E, Tsapas A, Dimitrakopoulos K, Kontzglou G, K Ionizakis I. Parotid gland oncocytoma: a case report. Cases J 2009;2:6423. Published online $2009 \mathrm{M}$ arch 10.

9. Diouf M S, Claros P, ClarosA. Oncocytoma of the parotid gland: a case report. Rev Laryngol Otol Rhinol (Bord) 2012;133(2): 109-112.

10. Chakrabarti I, Basu A, Ghosh N. Oncocytic lesion of parotid gland: a dilemma for cytopathologists. J Cytol 2012;29:80-82. 